\title{
The significance of lower extremity FDG PET/CT imaging in patients with unknown primary tumor
}

\author{
Bekir Tasdemir $^{1} *$, Zeki Dostbil ${ }^{1}$, Kemal Unal $^{2}$, Sule Yildirim ${ }^{3}$, Ayse Nur Akatli ${ }^{4}$
}

\begin{abstract}
If a suspicious finding for primary site of an unknown primary tumor (UPT) is found in limited whole-body FDG $\mathrm{PET} / \mathrm{CT}$ imaging area, imaging of lower extremities is generally not performed in routine practice. This approach may not be true. In this case, FDG PET/CT imaging was performed in patient with UPT. The limited whole-body FDG PET/CT images showed an increased FDG uptake in a thyroid nodule which was seemed to be a primary lesion at first sight. But similar FDG PET/CT findings might be observed in benign thyroid nodules. So we also acquired FDG PET/CT images of the lower extremities. Then, a mass showing increased FDG uptake was seen in the left thigh. On histopathologic examination, the thyroid nodule was found to be benign and the left thigh mass was diagnosed with a malignant (hemangiopericytoma). This case demonstrates contribution of lower extremity FDG PET/CT imaging to detection of primary site of UPTs in suspected situations
\end{abstract}

Key words: Positron emission tomography, unknown primary tumor, field of view, lower extremity, hemangiopericytoma

\section{Introduction}

Unknown primary tumor (UPT) is described as a proven malignity with unidentified primary origin (1). UPTs account for approximately $5 \%$ of all malignancies and it is the fourth most common cause of death from cancer $(2,3)$. Diagnosis of the primary origin is essential for the treatment of the patient. Nowadays, 2-(18F) fluoro-2-deoxy-D-glucose (FDG) positron emission tomography/computed tomography (PET/CT) whole-body imaging is widely used for this purpose (1). Even though FDG PET/CT is entitled as whole-body imaging, the scanning is routinely performed between the base of skull and upper-mid thigh (4). Nevertheless, it is the right approach to image true whole-body in case of tumors having high risk for the involvement of scalp, skull, brain, or lower extremities (5). In this report, we aimed to emphasize that the significance of adding lower extremity to the FDG PET/CT imaging area in patients with UPT

Case

The patient was a 60 -year-old female with complaints of cough, exhaustion, and weight loss for two months.
Thorax CT scan revealed multiple regularbordered and round-shaped bilateral pulmonary nodules, of which the largest one had a diameter of 15 $\mathrm{mm}$. The nodules had been thought to be metastatic, and FDG PET/CT scanning was performed for the metabolic characterization of the nodules and also for the diagnosis of a potential primary tumor. The limited whole-body FDG PET/CT images showed multiple bilateral pulmonary nodules, in which the largest one had a dimensions of $17 \times 15 \mathrm{~mm}$ and a maximum of standardized uptake value (SUVmax) of 4 (Figure 1). Also, an increased FDG uptake was seen in a regular-bordered and round-shaped left lobe thyroid nodule with the dimensions of $10 \times 10 \mathrm{~mm}$ and a SUVmax of 6.1 (Figure 2 and 3).

Although the thyroid nodule seemed to be a primary lesion at first sight, it was possible to observe similar FDG uptake patterns on PET/CT in benign thyroid nodules. Therefore, the FDG PET/CT images of the lower extremities were also acquired. A lobulated mass with millimetric calcifications between the posterior thigh muscles in the left lower extremity with the dimensions of $75 \times 54 \times 114 \mathrm{~mm}$ and increased heterogeneous FDG uptake with a SUVmax of 10 were seen (Figure 3 and 4).

Received: 02-07-2015, Accepted 14-07-2015, Available Online 01-10-2015

1Dicle University, Medical Faculty, Department of Nuclear Medicine, Diyarbakir, TURKEY

2Izmir University, Medical Faculty, Department of Nuclear Medicine, Izmir, TURKEY

3 Ankara Oncology Research and Training Hospital, Department of Nuclear Medicine, Ankara, TURKEY

4Inonu University, Medical Faculty, Department of Pathology, Malatya, TURKEY

*Corresponding Author: Bekir Tasdemir E-mail: drbkr@hotmail.com 
Fine needle aspiration biopsy was taken from the thyroid nodule. On thyroid fine needle aspiration cytology examination, although the aspiration material was hypocellular, the thyrocytes seen were benign. (Figure 5)
The thigh mass was gone to fine needle aspiration cytology first and it was excised totally. Then it was diagnosed histopathologically as intermediate grade malignant soft tissue sarcoma in the structure of hemangiopericytoma (Figure 6).

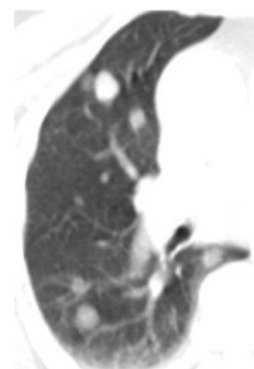

A

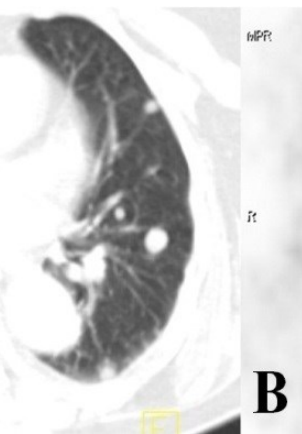

B

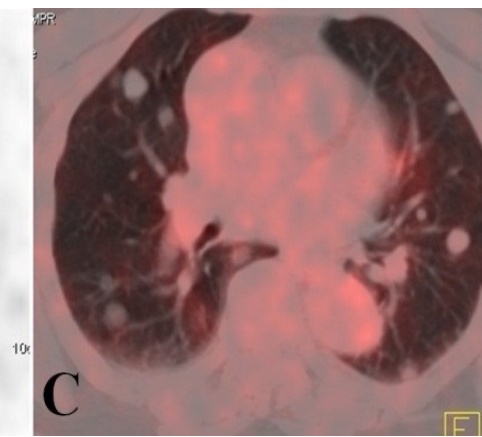

Figure 1. Multiple bilateral pulmonary nodules on axial CT (A), PET (B) and PET/CT (C) images.

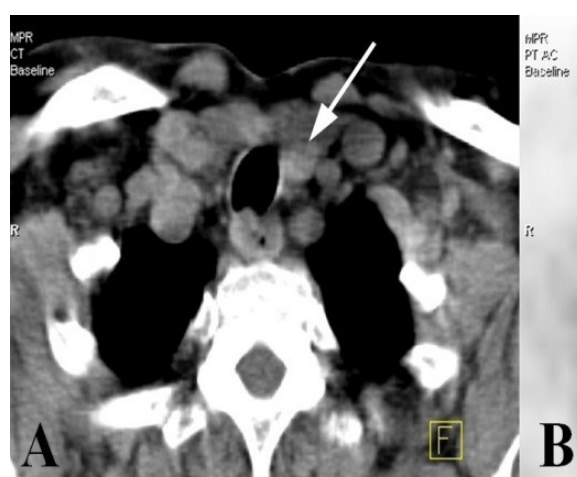

Figure 2. Thyroid nodule in the left lobe on axial CT (A), PET (B) and PET/CT (C) images (arrows).

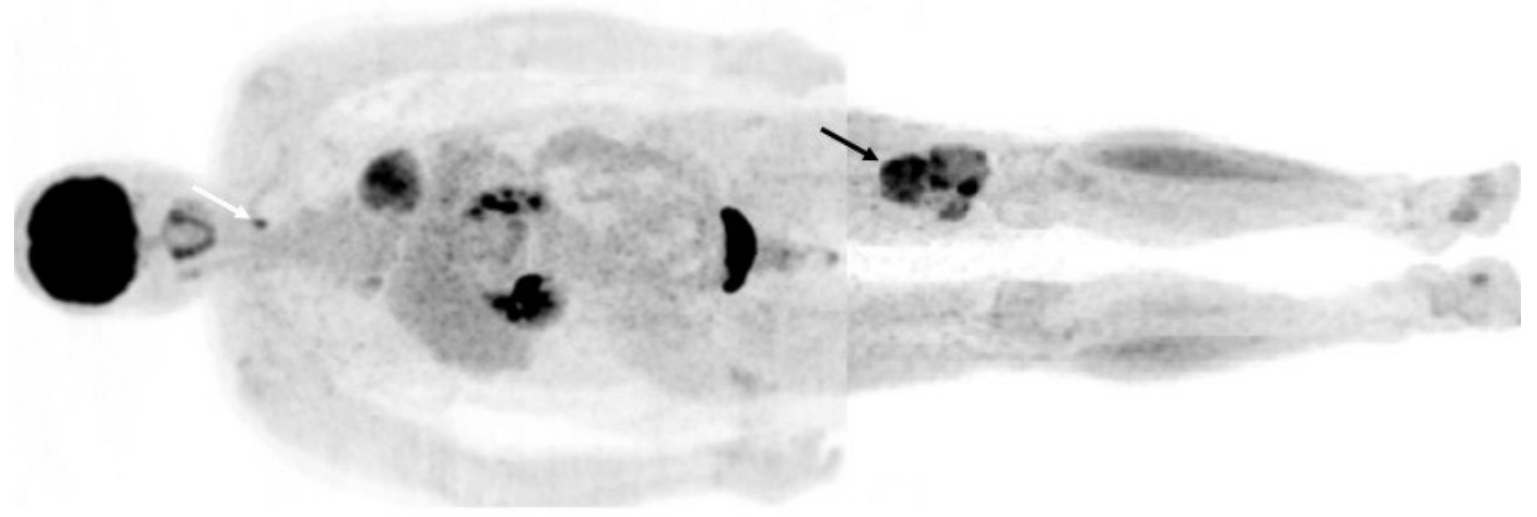

Figure 3. Maximum intensity projection (MIP) image of the patient. White arrow shows thyroid nodule in the left lobe and black arrow shows lobulated mass between the posterior thigh muscles in the left lower extremity. 


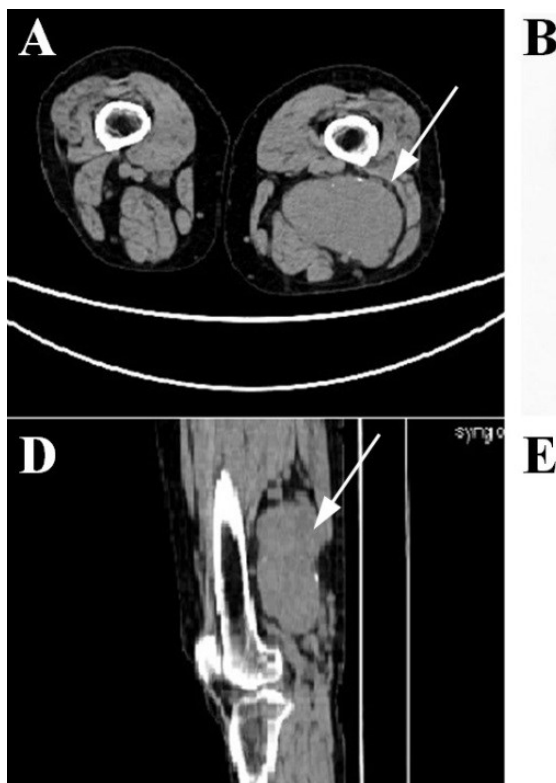

B

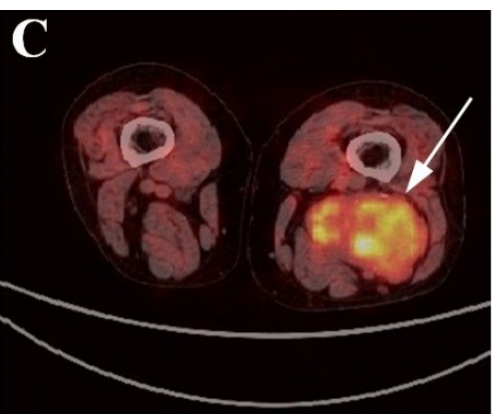

Figure 4. Lobulated mass between the posterior thigh muscles in the left lower extremity on axial CT (A), PET (B), PET/CT (C) and sagittal CT (D), PET (E) and PET/CT (F) images

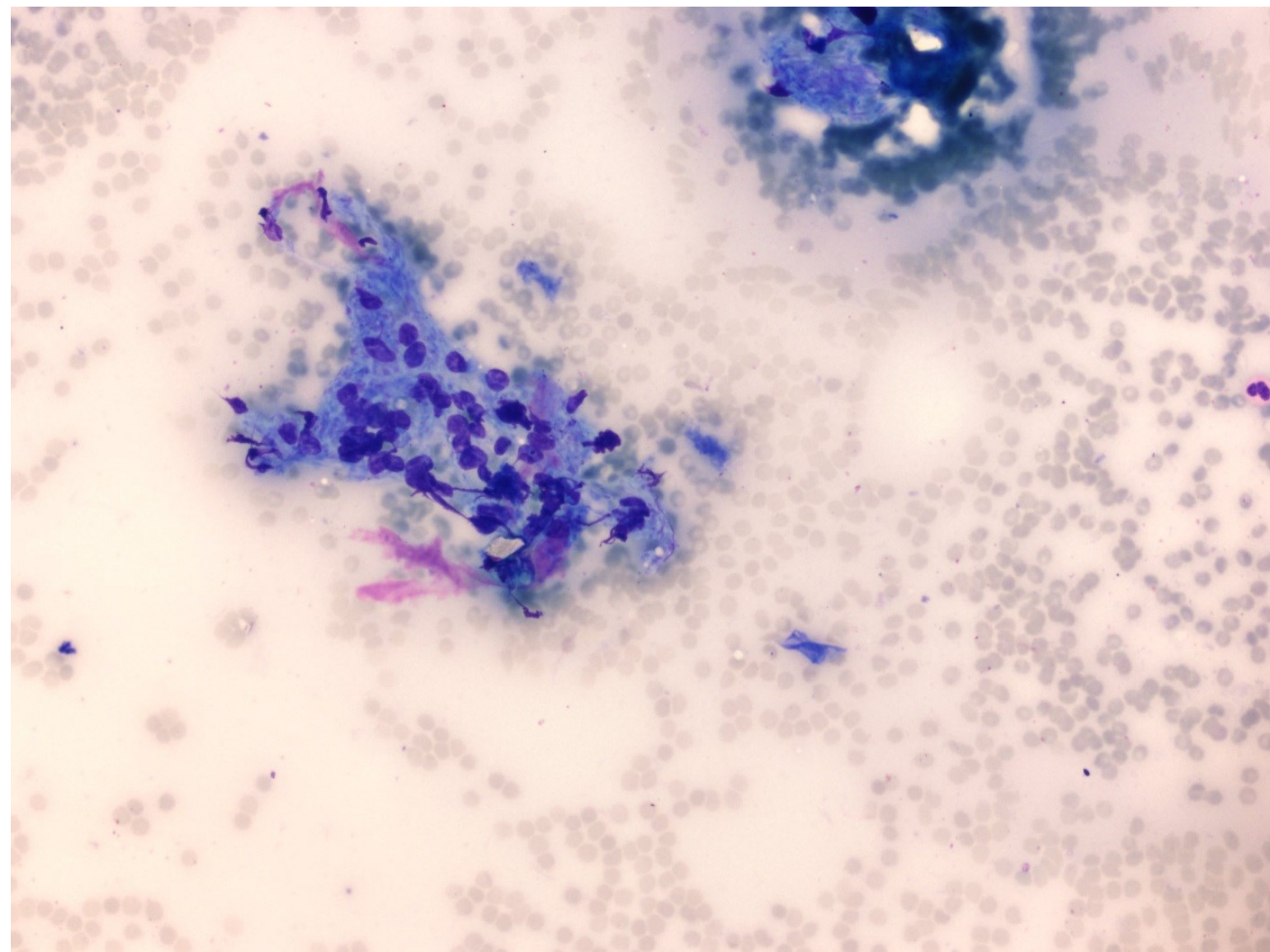

Figure 5. Benign-looking thyrocytes. 


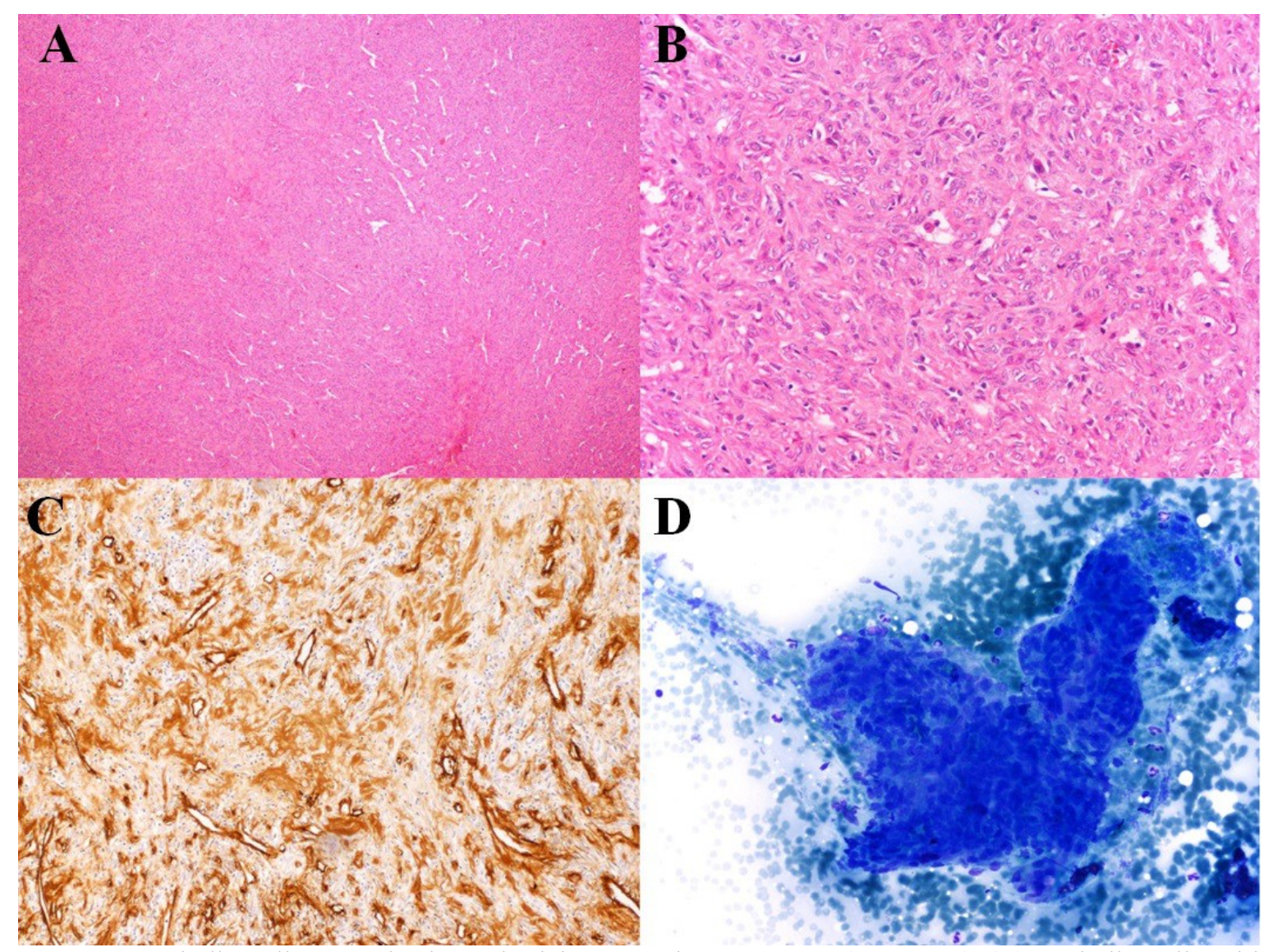

Figure 6. Spindle cell tumor having arborizing vascular structures $(H \& E, \times 40)(A)$. Spindle cells with moderately cellular tumor $(\mathrm{H} \& \mathrm{E}, \times 200)(\mathrm{B})$. Tumor cells stained with Factor VIII antibody (Factor VIII, x100) (C). Cohesive spindle-shaped cell cluster on cytology (MGG, x200) (D).

\section{Discussion and Conclusion}

In literature, there are several reports about the inclusion of other body parts to the limited wholebody area on FDG PET/CT imaging (4,6-13). However, only two of them have evaluated the clinical contributions of the lower extremity imaging $(4,9)$. In the study performed by Osman et al., the lower extremity imaging did not result in any change in terms of the stage of the disease in 14 patients with UPT (4). Sebro et al. studied 46 patients with UPT and reported that the lower extremity scanning changed the tumor stage in only one patient (9).

In our literature review, we did not identify any study that has revealed any primary origin by means of the lower extremity FDG PET/CT scanning in patients with UPT. Besides, there is no clear statement in the main FDG PET/CT guidelines about including the lower extremities in FDG PET/CT imaging in patients with UPT $(5,14,15)$. For this reason, there are various implementations in the nuclear medicine clinics and there is no standard imaging procedure for this indication.
Some clinics perform limited whole-body scanning, paying no attention to the detection of a primary lesion. And, some clinics include other body parts if a primary tumor is not detected in limited whole-body field of view. If a suspicious primary lesion is found in the limited whole-body scan, then imaging of other body parts are generally not performed. This case represents that this kind of approach may not be true.

In conclusion, this case demonstrates the contribution of adding lower extremity to the limited whole-body imaging area on FDG PET/CT imaging for the detection of the primary site of UPT in case primary tumor is not found or the determined primary focus has any doubt in the limited whole-body imaging area

Conflict of Interest: The authors declare no potential conflicts of interest with respect to the research, authorship, and/or publication of this article. We thank Ahmet Yilmaz from Dicle University Medical Faculty for his invaluable help the manuscript's publishing process. 


\section{References}

1. Park JS, Yim JJ, Kang WJ, et al. Detection of primary sites in unknown primary tumors using FDG-PET or FDGPET/CT. BMC Res Notes 2011;4:56

2. Fizazi K, Culine S. Metastatic carcinoma of unknown origin. Bull Cancer 1998;85:609-617.

3. Kwee TC, Basu S, Cheng G, Alavi A. FDG PET/CT in carcinoma of unknown primary. Eur J Nucl Med Mol Imaging 2010;37:635-644.

4. Osman MM, Chaar BT, Muzaffar R, et al. 18F-FDG PET/CT of patients with cancer: comparison of wholebody and limited whole-body technique. AJR Am J Roentgenol 2010;195:1397-1403.

5. Delbeke D, Coleman RE, Guiberteau MJ, et al. Procedure guideline for tumor imaging with 18F-FDG PET/CT 1.0. J Nucl Med 2006;47:885-895.

6. Abdelmalik AG, Alenezi S, Muzaffar R, Osman MM. The Incremental Added Value of Including the Head in (18)FFDG PET/CT Imaging for Cancer Patients. Front Oncol $2013 ; 3: 71$

7. Lee HY, Lee KS, Kim BT, et al. Diagnostic efficacy of $\mathrm{PET} / \mathrm{CT}$ plus brain MR imaging for detection of extrathoracic metastases in patients with lung adenocarcinoma. J Korean Med Sci 2009;24:1132-1138.

Nguyen NC, Chaar BT, Osman MM. Prevalence and patterns of soft tissue metastasis: detection with true whole-body F-18 FDG PET/CT. BMC Med Imaging 2007;7:8.
9. Sebro R, Mari-Aparici C, Hernandez-Pampaloni M. Value of true whole-body FDG PET/CT scanning protocol in oncology: optimization of its use based on primary diagnosis. Acta Radiol 2013;54:534-539

10. Bochev P, Klisarova A, Kaprelyan A, et al. Brain metastases detectability of routine whole body (18)F-FDG PET and low dose CT scanning in 2502 asymptomatic patients with solid extracranial tumors. Hell J Nucl Med 2012;15:125-129.

11. Kitajima K, Nakamoto Y, Okizuka H, et al. Accuracy of whole-body FDG PET/CT for detecting brain metastases from non-central nervous system tumors. Ann Nucl Med 2008;22:595-602.

12. Rohren EM, Provenzale JM, Barboriak DP, Coleman RE. Screening for cerebral metastases with FDG PET in patients undergoing whole-body staging of non-central nervous system malignancy. Radiology 2003;226:181-187.

13. Posther KE, McCall LM, Harpole DH Jr, et al. Yield of brain $18 \mathrm{~F}-\mathrm{FDG}$ PET in evaluating patients with potentially operable non-small cell lung cancer. J Nucl Med 2006;47:1607-1611.

14. Boellaard R, O'Doherty MJ, Weber WA, et al. FDG PET and PET/CT: EANM procedure guidelines for tumor PET imaging: version 1.0. Eur J Nucl Med Mol Imaging 2010;37:181-200.

15. ACR-SPR practice guideline for performing FDG PET/CT in oncology. American College of Radiology Web site. http://www.acr.org/ /media/71B746780F934F6D8A1BA5 CCA5167EDB.pdf Accessed 06 Jan 2015.

Copyright (C) 2014 The Author(s); This is an open-access article distributed under the terms of the Creative Commons Attribution License (http://creativecommons.org/licenses/by/4.0), which permits unrestricted use, distribution, and reproduction in any medium, provided the original work is properly cited. All Rights reserved by international journal of Medical Science and Discovery. 